Supporting Information

\title{
Unravelling the Structured Solvation Shell of Zwitterion Nanoparticles for Controlled Release of Nitric Oxide
}

Sungwon Jung§, Kyungtae Park§, Sohyeon Park, Jiwoong Heo, Woojin Choi, and Jinkee Hong*

$\S$ S.J. and K.P. contributed equally to this paper.

School of Chemical \& Biomolecular Engineering, Yonsei University, 50 Yonsei-ro, Seodaemun-gu, Seoul, 03722, Republic of Korea

*E-mail: jinkee.hong@yonsei.ac.kr 
a

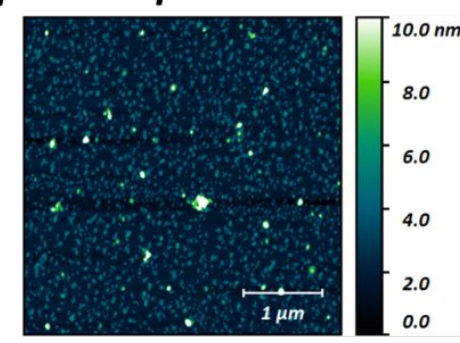

$\boldsymbol{b}$

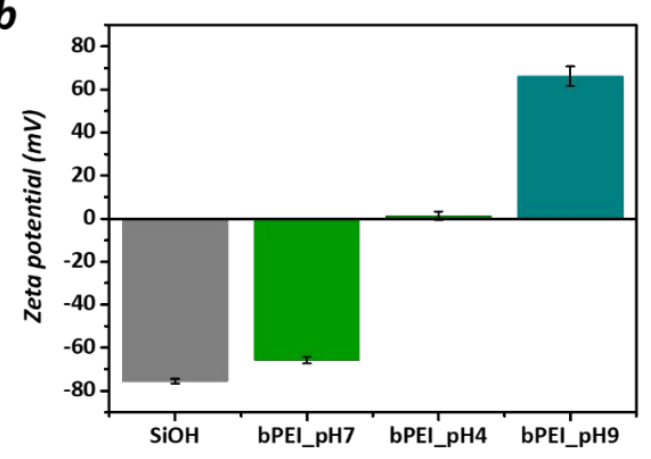

pH 7

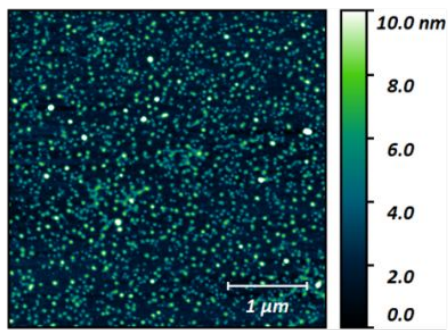

C

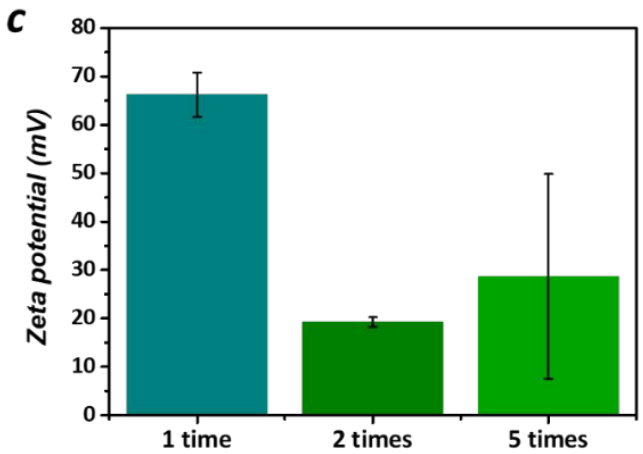

Figure. S1| Pre-experiment data for optimum coating on silica nanoparticles. (a) Atomic force microscopy (AFM) image of different $\mathrm{pH}$ of bPEI-coated flat substrates. (b) Zeta potential data of different $\mathrm{pH}$ of bPEI coated on nanoparticles. (c) Zeta potential data according to the number of bPEI coated on silica nanoparticles. 
1,3-propane sultone, PS

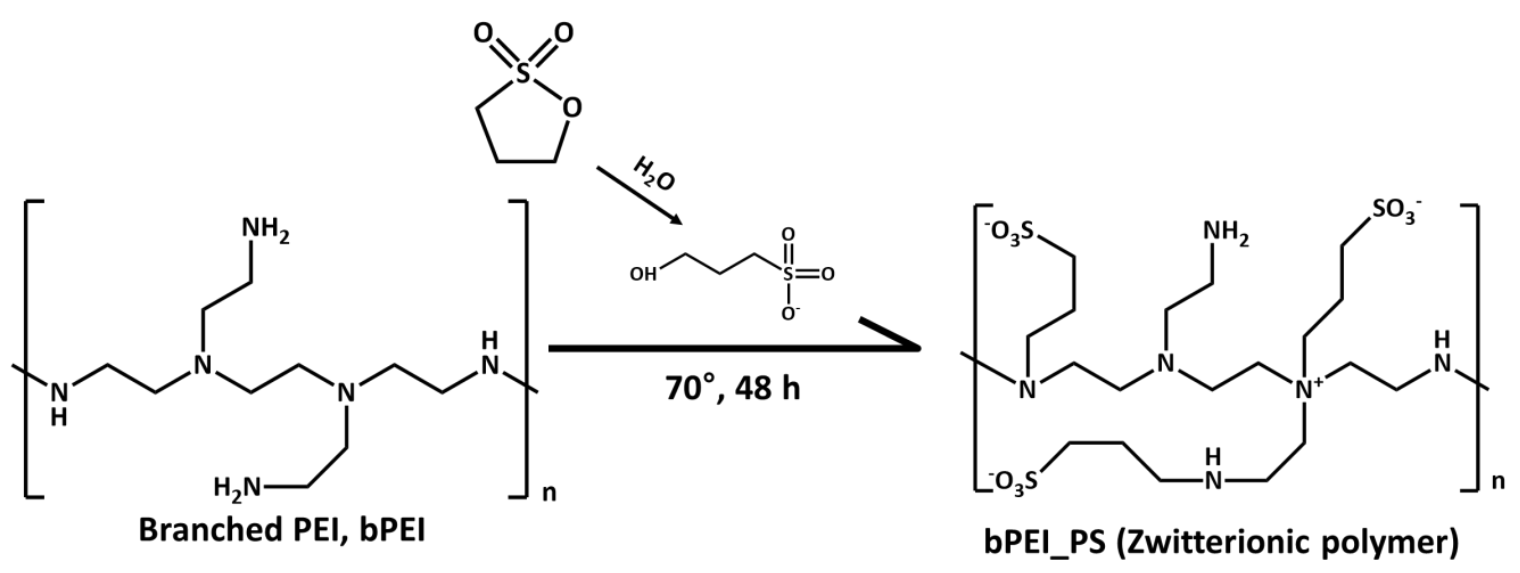

Figure. S2| Chemical reaction mechanism of bPEI react with 1,3-propane sultone (PS) 


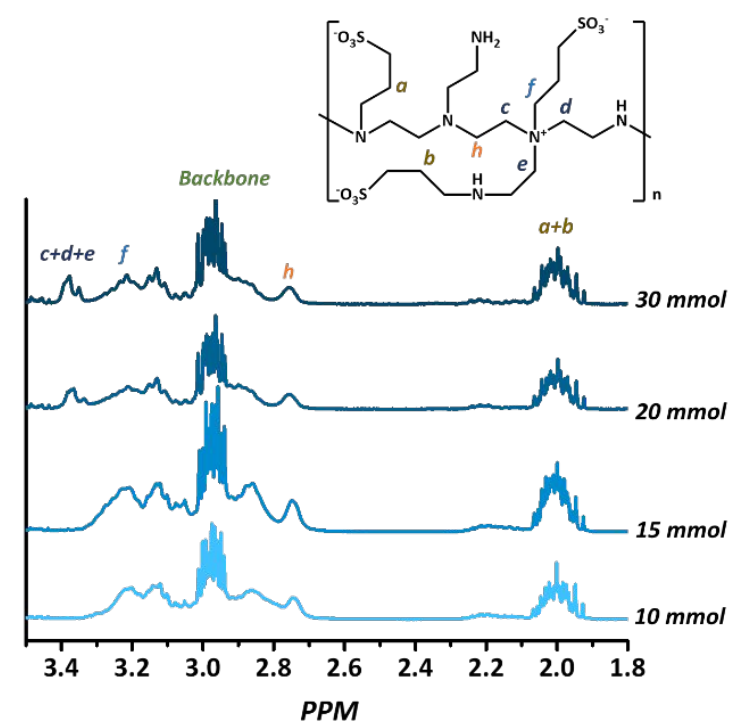

\begin{tabular}{|c|c|c|c|c|}
\hline & $10 \mathrm{mmol}$ & $15 \mathrm{mmol}$ & $20 \mathrm{mmol}$ & $30 \mathrm{mmol}$ \\
\hline Prim+Sec & 0.28 & 0.29 & 0.37 & 0.39 \\
\hline Tertiary & 0.48 & 0.49 & 0.48 & 0.47 \\
\hline Quaternary & $x$ & $x$ & 0.13 & 0.14 \\
\hline Backbone & 1 & 1 & 1 & 1 \\
\hline
\end{tabular}

Figure. S3| Nuclear magnetic resonance (NMR) spectroscopy analysis of bPEI_PS polymer with different concentration of PS 

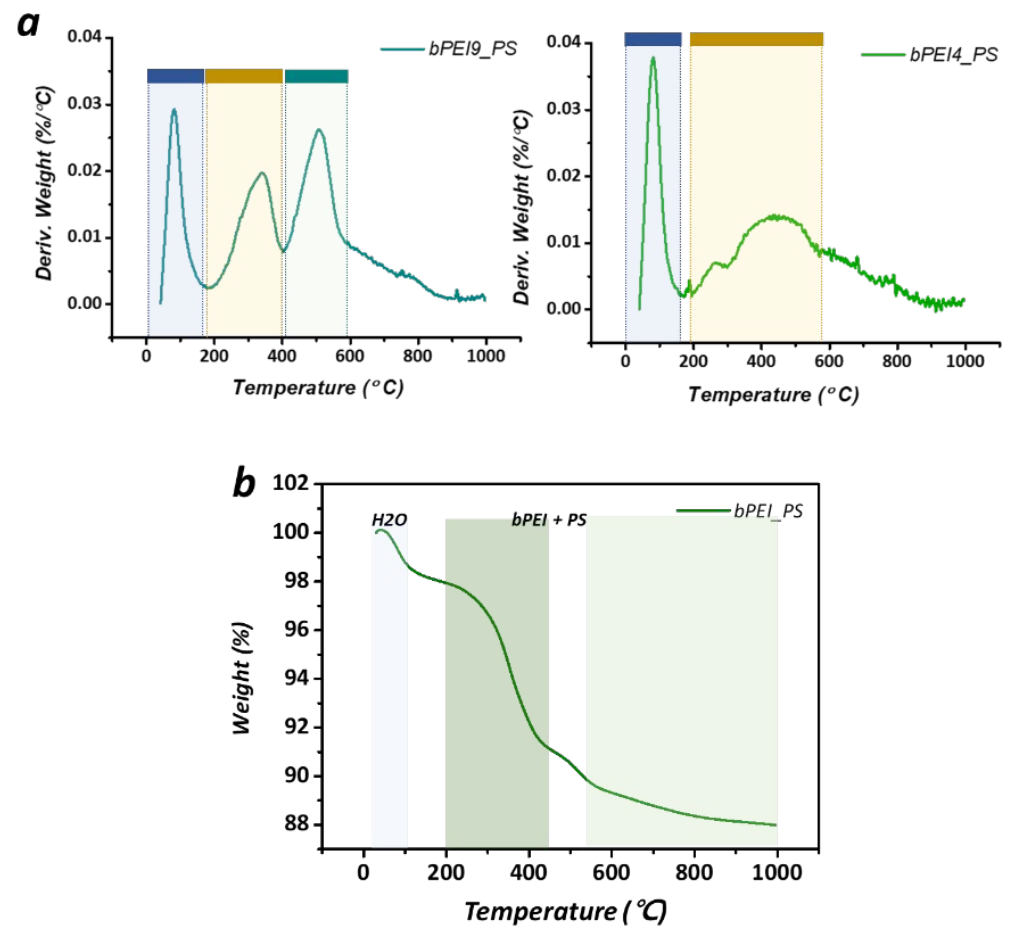

Figure. S4| Thermogravimetric analysis (TGA) of ZWNP (a) Derivative weight curve based on different $\mathrm{pH}$ of bPEI ( $\mathrm{pH} 4$ and $\mathrm{pH}$ 9). (b) Weight-temperature curve of ZWNP coated with bPEI_PS (pH 9). 

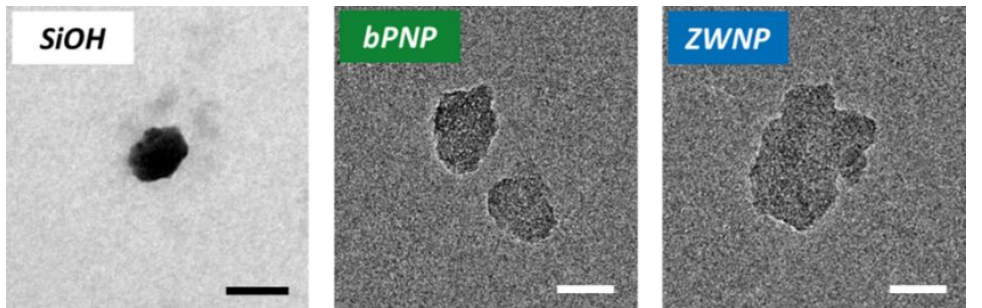

Figure. S5| Transmission electron microscopy (TEM) images of SiOH, bPNP, ZWNP and NO_ZWNP (scale: $100 \mathrm{~nm}$ ). 

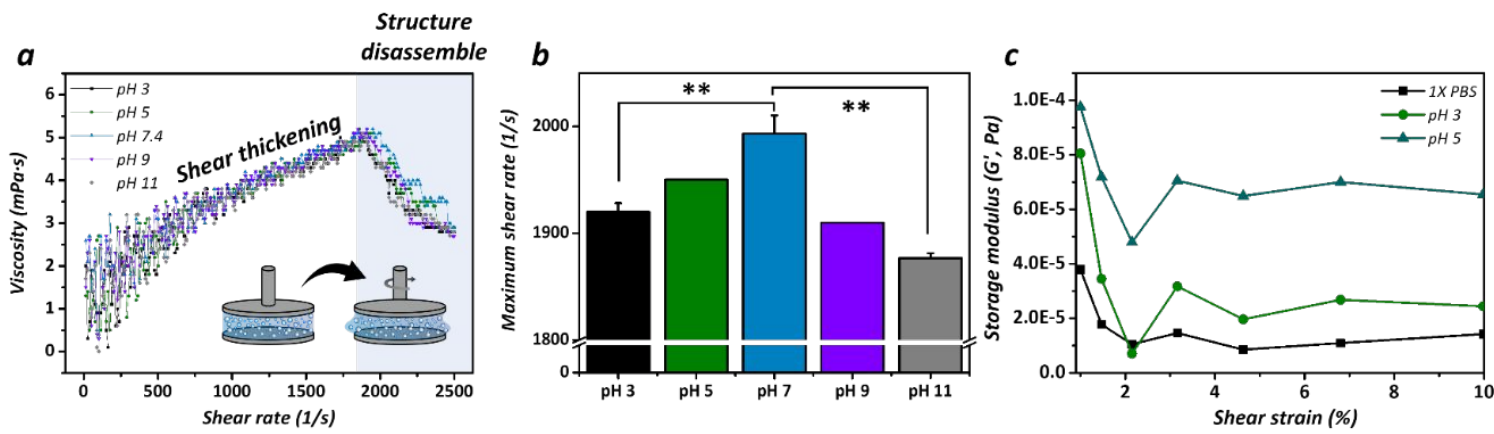

Figure. S6| Rheological analysis of highly concentrated ZWNP solution (solvent as 1X PBS).

(a) Shear-rate sweep of ZWNP solution from 0 to 2500 1/s. (b) Endurable maximum shear rate of high-concentration of ZWNP solution with different $\mathrm{pH}$. (c) Storage modulus (G') of ZWNP strain from 1 to $10 \%$. 

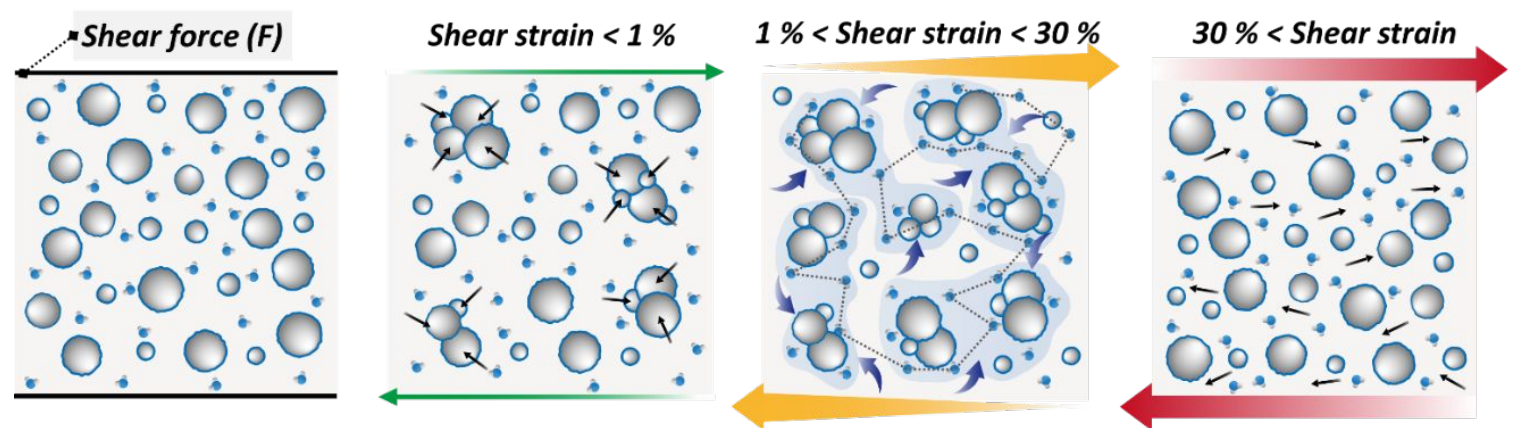

Figure. S7| Illustration of ZWNP and water molecule behavior depend on shear strain. 

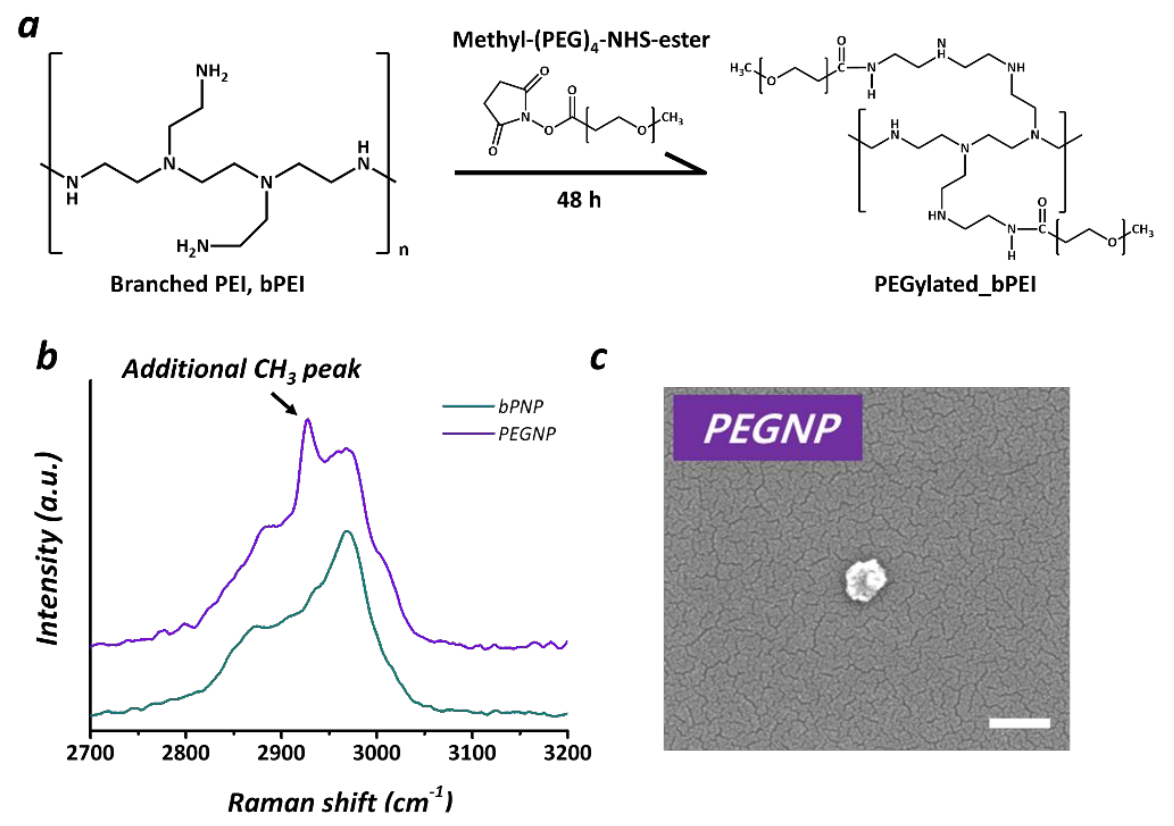

Figure. S8 $\mid$ Detailed information of PEGNP. (a) Reaction mechanism for PEGylated bPEI, (b) raman spectroscopy data of PEGNP, (c) SEM image of PEGNP. 

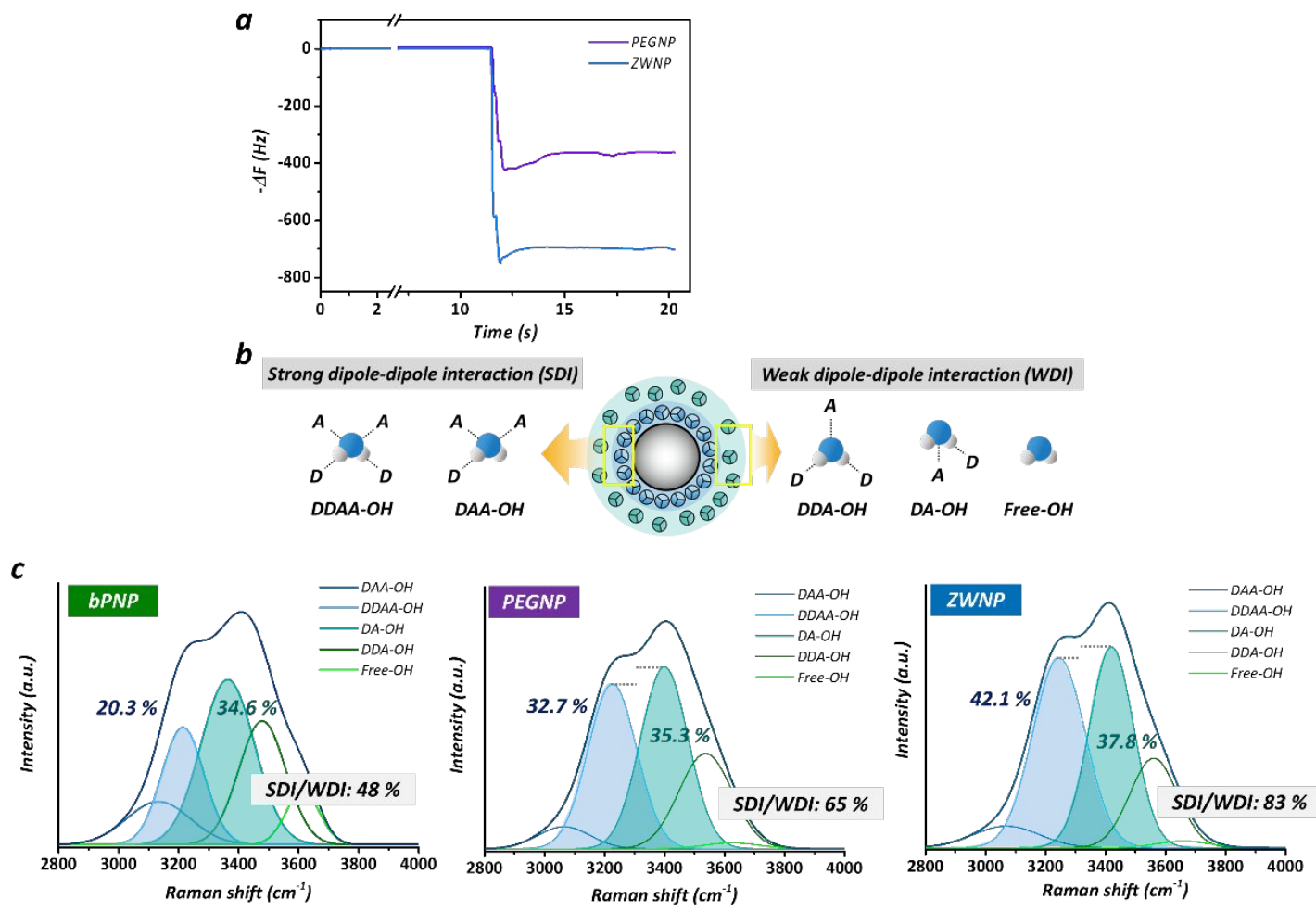

Figure. S9| Characterization of PEGylated bPNP (PEGNP) for comparing with ZWNP. (a) QCM analysis of water molecule absorption on PEGNP coated electrode compared to ZWNP coated electrode. (b) Illustration of dipole-dipole interaction (DI) of water molecules. DI of water can be separated to strong dipole-dipole interaction (SDI) and weak dipole-dipole interaction (WDI) depending on the number of donor and acceptor interactions. (c) Inspection of ratio of SDI and WDI by raman spectroscopy of solvation shell of PEGNP (left) and ZWNP (right). 


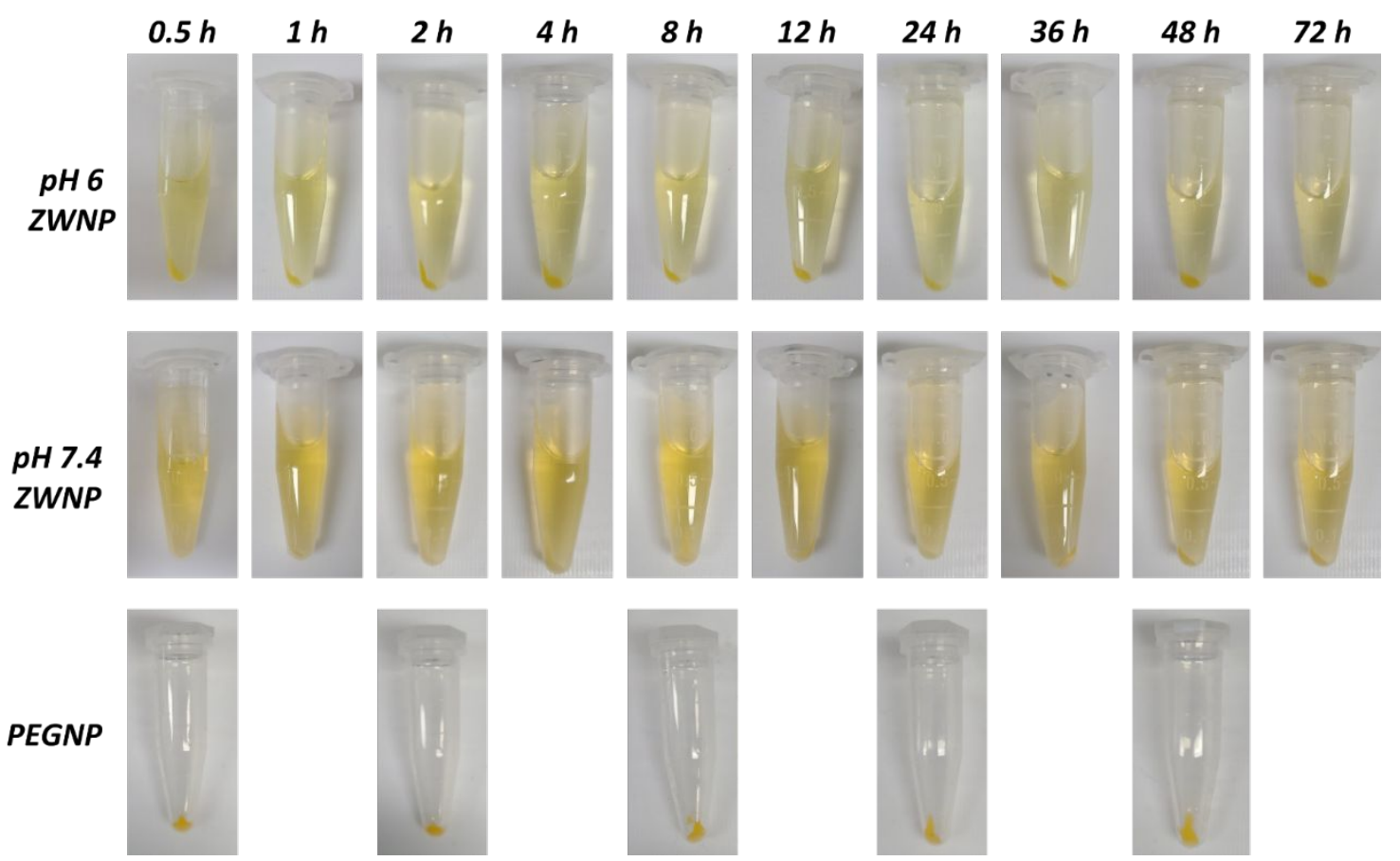

Figure. S10| Antifouling effect of ZWNP (with different $\mathrm{pH}$ ) and PEGNP at each time point 


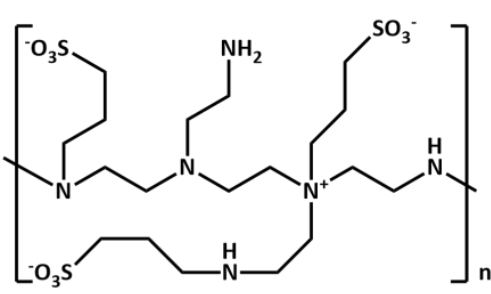

bPEI_PS (Zwitterionic polymer)

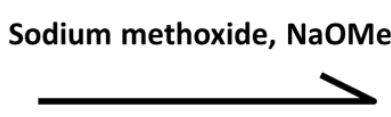

10 bar, $72 \mathrm{~h}$

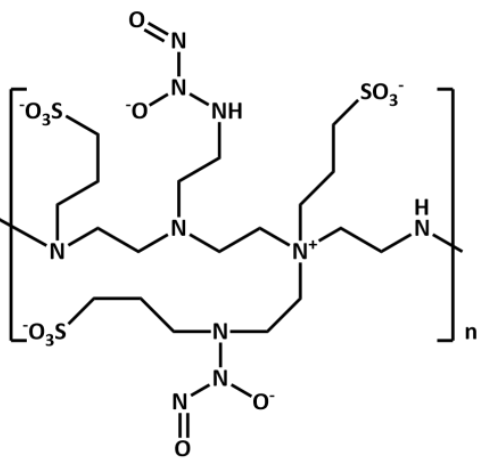

NONOate_bPEI_PS

Figure. S11| Reaction mechanism for NONOate_bPEI_PS 
$a$

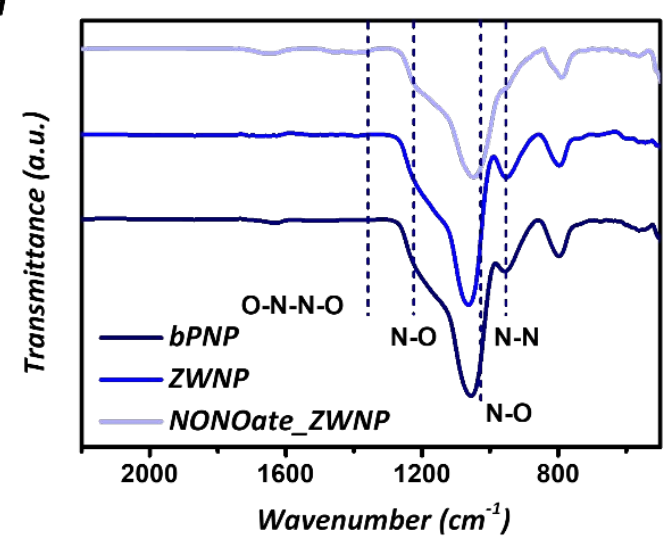

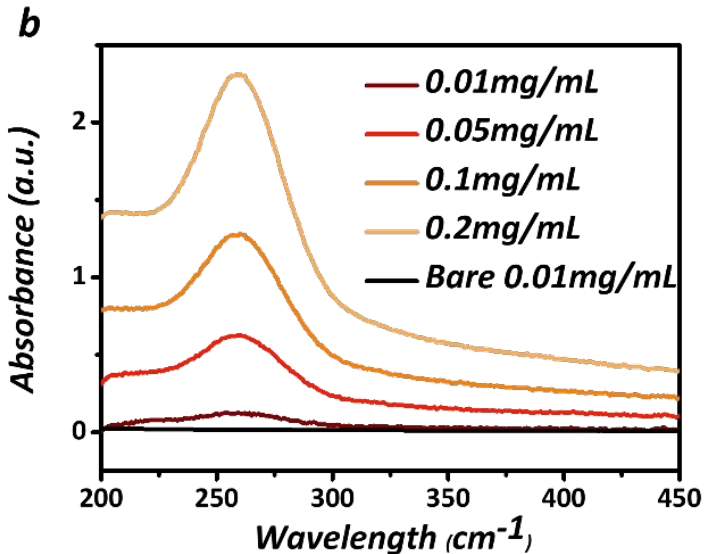

Figure. S12| (a) FT-IR analysis for ZWNP before and after high-pressure reaction. (b) UV-vis analysis of NO_ZWNP with different concentrations. 


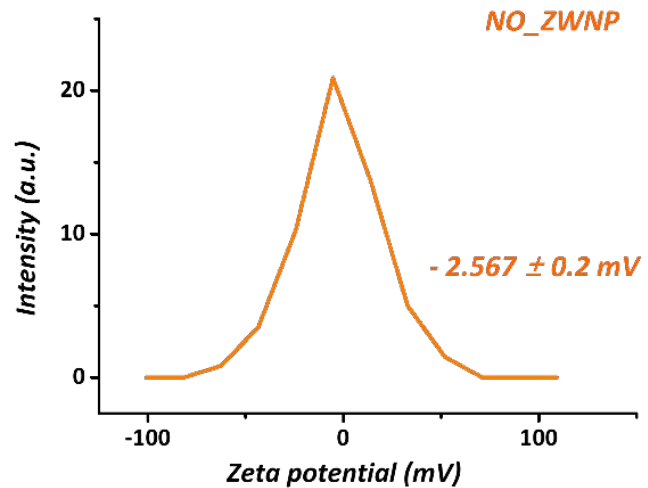

Figure. S13 Zeta potential results of ZWNP after high-pressure reaction 

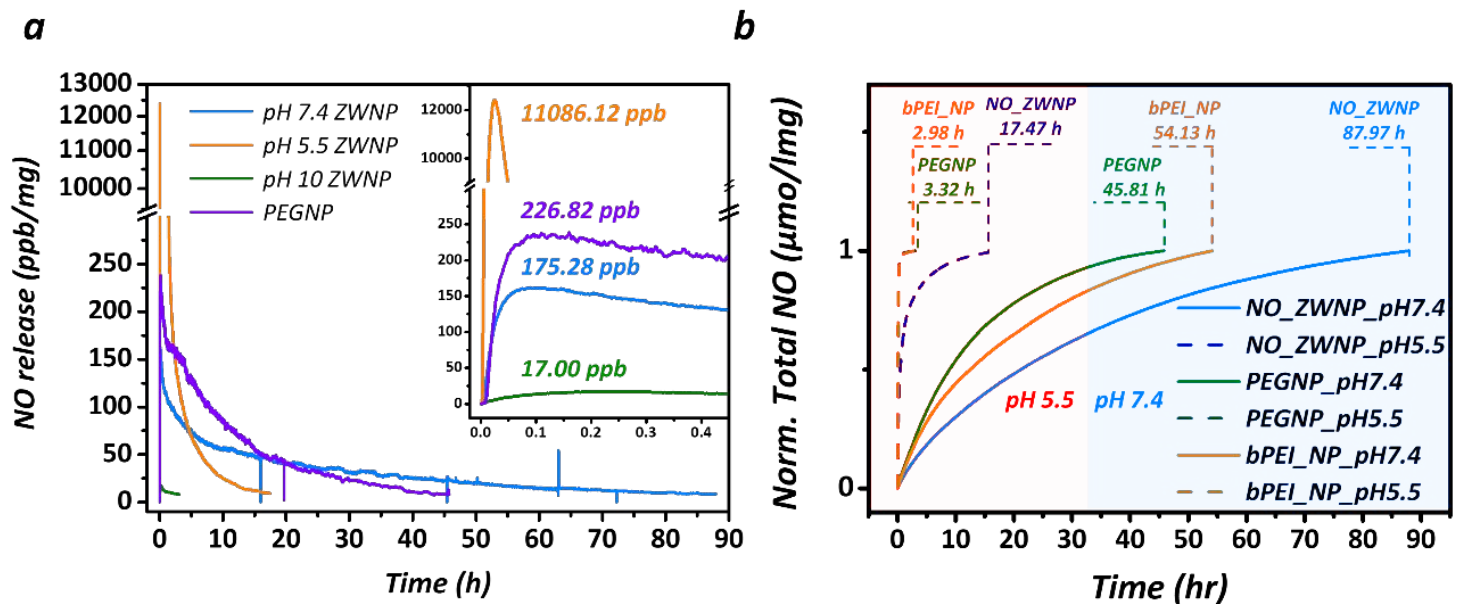

Figure. S14| (a) Magnified ZWNP's NO release profile with different $\mathrm{pH}$ conditions. (b) Comparison of the bPEI_NP, PEGNP and NO_ZWNP in pH 5.5 and pH 7.4 condition. 

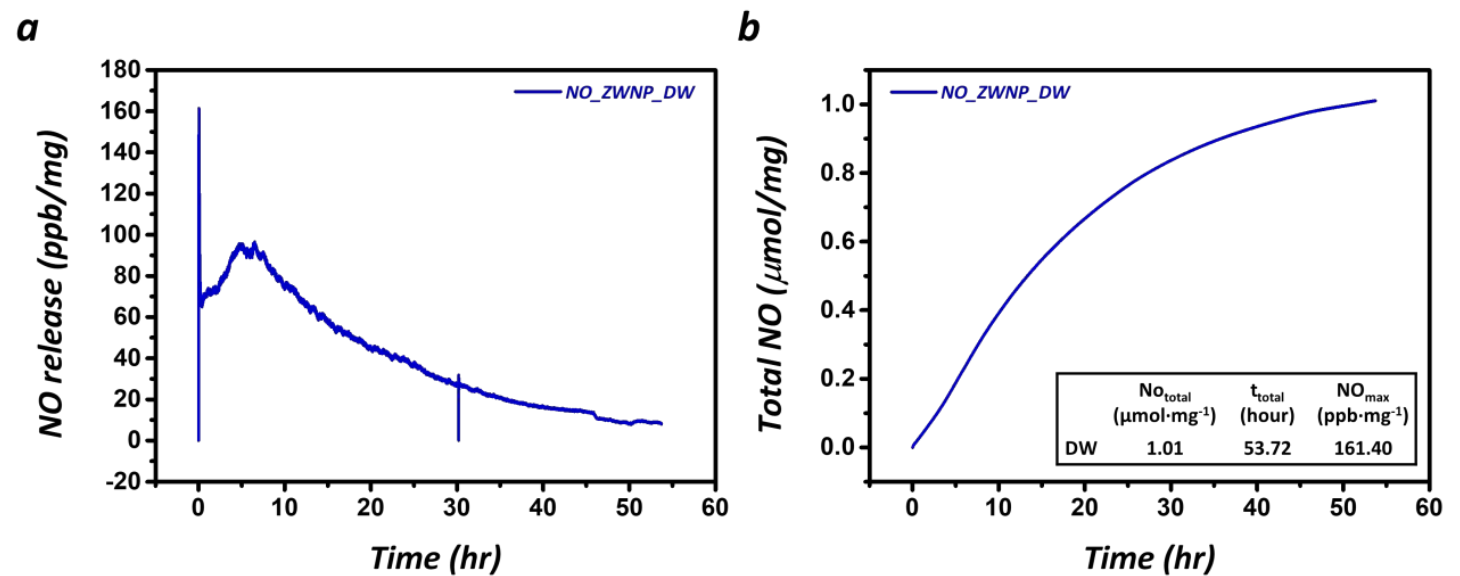

Figure. S15| bPEI coated nano particle's NO release profile after NO donor synthesis. (a) real time NO release kinetics (b) accumulated NO release amount

$\mathrm{NO}_{\text {total }}\left(\mu \mathrm{mol} \cdot \mathrm{mg}^{-1}\right)$ : Total amount of $\mathrm{NO}$ released from the particles, $\mathrm{NO}_{\max }\left(\mathrm{ppb} \cdot \mathrm{mg}^{-1}\right)$ : Maximum amount of NO from the release profile, $t_{\text {total }}(\mathrm{min})$ : Duration time of total release, Analysis condition: $1 \mathrm{X} \mathrm{PBS}, \mathrm{pH} 7.4,37^{\circ} \mathrm{C}$ 

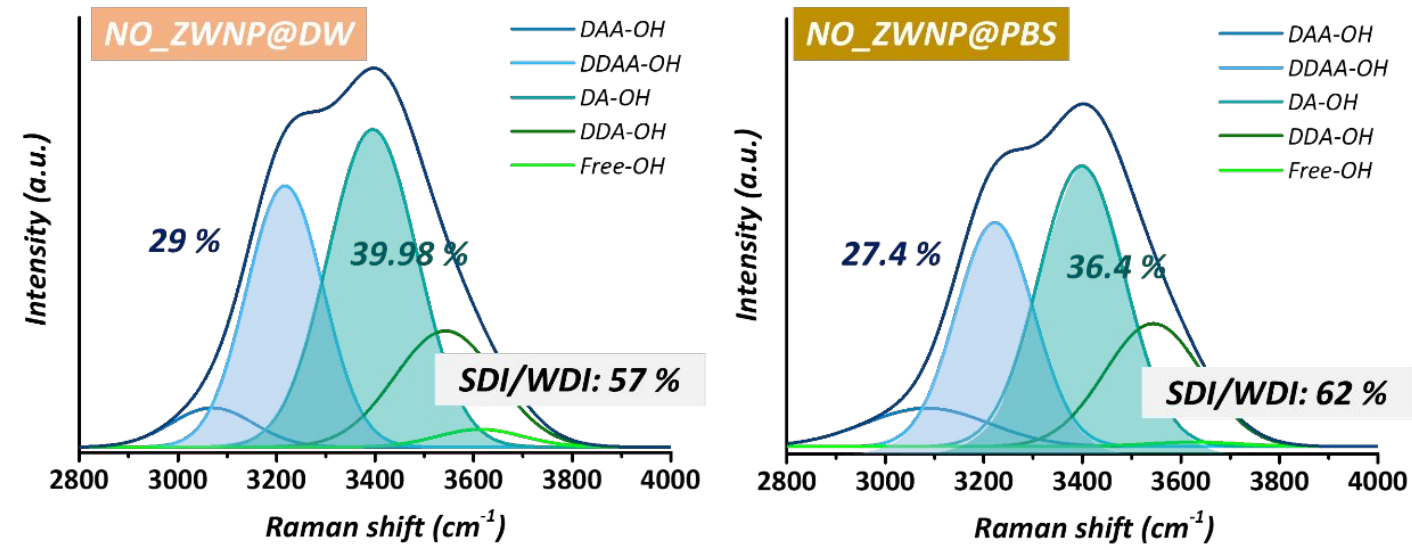

Figure. S16| Inspection of ratio of SDI and WDI by raman spectroscopy of solvation shell of ZWNP after high-pressure reaction in DW and PBS environment. 

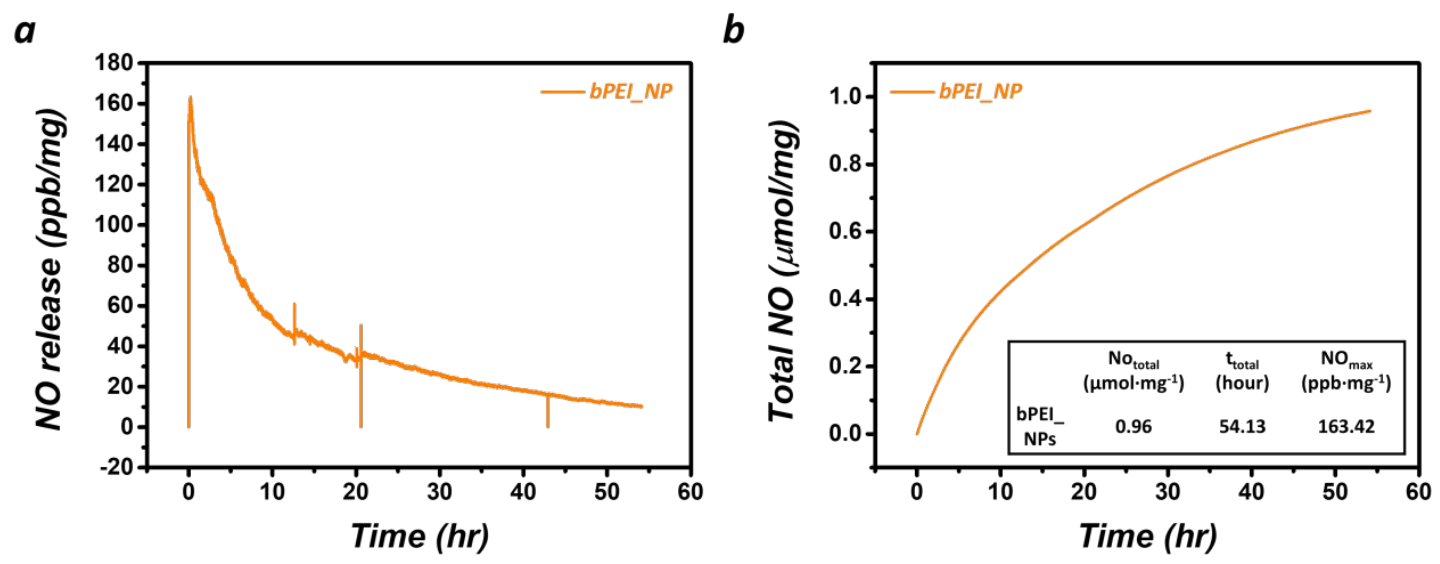

Figure. S17| ZWNP's NO release profile in deionized water condition. (a) real time NO release kinetics (b) accumulated NO release amount

$\mathrm{NO}_{\text {total }}\left(\mu \mathrm{mol} \cdot \mathrm{mg}^{-1}\right)$ : Total amount of $\mathrm{NO}$ released from the particles, $\mathrm{NO}_{\max }\left(\mathrm{ppb} \cdot \mathrm{mg}^{-1}\right)$ : Maximum amount of NO from the release profile, $t_{\text {total }}(\mathrm{min})$ : Duration time of total release, Analysis condition: $1 \mathrm{X}$ PBS, $\mathrm{pH} 7.4,37^{\circ} \mathrm{C}$ 

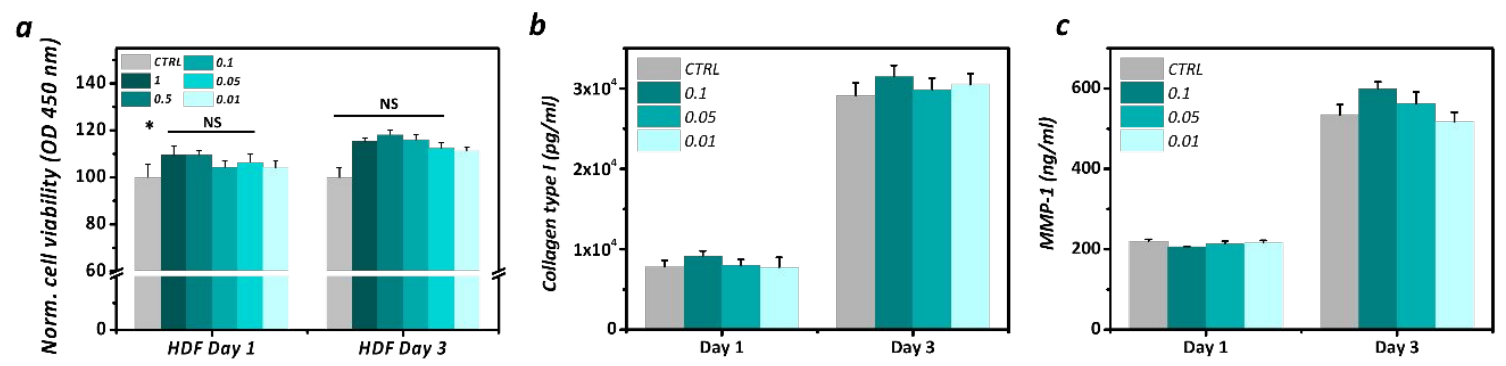

Figure. S18| (a) In vitro cytotoxicity test of ZWNP with various concentration for HDF. (b, c) Quantitative analysis of collagen synthesis and MMP-1 secretion from HDF after treatment of ZWNP via enzyme-linked immunosorbent assay (ELISA). Both ELISA data were statistically nonsignificant and are not indicated in the figure. ${ }^{*} \mathrm{P}<0.05, * * \mathrm{P}<0.01,{ }^{*} * * \mathrm{P}<0.001$, $* * * \mathrm{P}<0.0001$ ( $\mathrm{t}$-test, all experiments were biologically independent) 


\section{Abbreviation list}

ZWNPs: sulfobetaine zwitterion nanoparticles

NO: nitric oxide

PI: isoelectric point

PEG: polyethylene glycol

OH: hydroxyl groups

bPEI: branched-polyethyleneimine

bPNP: branched-polyethyleneimine silica particles

PS: 1,3-propane sultone

PEGNP: PEGylated nanoparticle

NONOates: N-Diazeniumdiolates

NO_ZWNP: synthesized NO releasing ZWNP

SF-ZWNP: solvation shell formed-ZWNP

SiOH: silica nanoparticles

NF: non-freezable water

FB: freezable water

FW: free water

SDI: strong dipole-dipole interaction

WDI: weak dipole-dipole interaction

VEGF: vascular endothelial growth factor

MMP-1: matrix metalloproteinase-1 